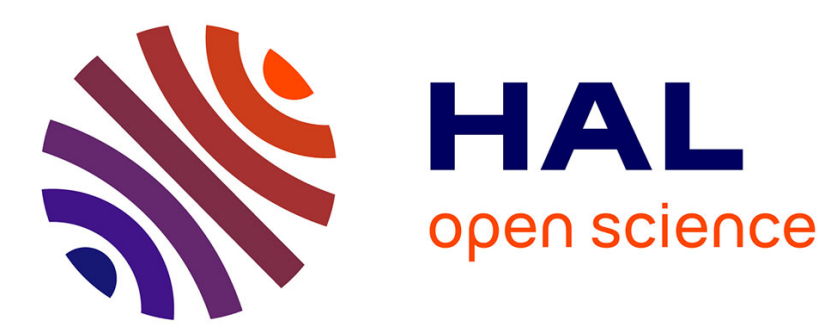

\title{
A computer aided education tool for electromagnetic scattering phenomena
}

\author{
Frédéric Thollon, Laurent Nicolas
}

\section{To cite this version:}

Frédéric Thollon, Laurent Nicolas. A computer aided education tool for electromagnetic scattering phenomena. IEEE Transactions on Magnetics, 2000, 36 (4 Part 1), pp.880-883. hal-00141508

\section{HAL Id: hal-00141508 \\ https://hal.science/hal-00141508}

Submitted on 18 Apr 2007

HAL is a multi-disciplinary open access archive for the deposit and dissemination of scientific research documents, whether they are published or not. The documents may come from teaching and research institutions in France or abroad, or from public or private research centers.
L'archive ouverte pluridisciplinaire HAL, est destinée au dépôt et à la diffusion de documents scientifiques de niveau recherche, publiés ou non, émanant des établissements d'enseignement et de recherche français ou étrangers, des laboratoires publics ou privés. 


\title{
A Computer Aided Education Tool for Electromagnetic Scattering Phenomena
}

\author{
Frederic Thollon and Laurent Nicolas
}

\begin{abstract}
In this paper, a computer aided education tool to understand electromagnetic scattering phenomena is described. "Real time" solving is performed using the finite element method coupled with a first order absorbing boundary condition. Computed results are displayed as a time animation. Three examples are presented: radiation from a wire (in order to illustrate propagation phenomena), scattering by a lossy dielectric cylinder (in order to compare induced and displacement currents) and scattering by a parallel plate waveguide (in order to understand the cutoff phenomenon).
\end{abstract}

Index Terms-Education, electromagnetic propagation, finite elements methods, scattering.

\section{INTRODUCTION}

$\mathbf{T}$ HE UNDERSTANDING of phenomena encountered in electrical engineering is facing the problem of using abstract notions like charges, fields or induced currents in materials. Furthermore, these notions are manipulated using even more abstract vectors or differential operators. This is one of the main reasons why most students in the field of electrical engineering do not have a deep understanding of the basic principles, even if they are able to solve standard problems. One way to prevent this misunderstanding is to provide them with easy to use, easy to implement packages having the following features:

- The results are mainly given in a graphical manner in order to provide the students with images "with which they can think about."

- The students can try many configurations in order to compare them and to understand the influence of different parameters on the phenomena. They can choose actually geometrical dimensions and physical properties. The students must have an "immediate" response to their questions: any interaction of the user with the keyboard or with the mouse requires a modification of the displayed results in real time.

- If necessary, animation has to be used in order to illustrate time dependent phenomena.

The second point is the main key point. This approach is different from the pure animation of solution of already solved problem as presented in [1], where the learning process is strongly guided. With our approach, the student is able to build actually her/his own thinking process: it is actually an inductive

Manuscript received October 25, 1999.

The authors are with CEGELY, UPRESA CNRS 5005, Ecole Centrale de Lyon, BP 163, 69131 Ecully cedex, France (e-mail: \{thollon; laurent $\} @$ eea.eclyon.fr).

Publisher Item Identifier S 0018-9464(00)06970-3. learning based on the intuitive understanding of the physical phenomena.

Taking into account these features, designing such applications leads to making several choices, depending on the illustrated physical phenomenon. First, the input geometric or physical parameters and the range in which they can vary have to be chosen. Second, it is necessary to choose the pertinent quantities to be visualized. Thirdly, the most efficient solving method has to be chosen in order to minimize as much as possible the solving time. On the other hand, since the results are mainly graphical, the accuracy of the solution is actually not very important.

We have already presented such computer aided education (CAE) tools [2]. Depending on the objective which is sought, they are based either on an analytical solution or on a finite element (FE) solution. In this paper, a new application-Depind - is presented. The studied problem is the scattering of a wave radiated from different types of sources by an object of infinite length with a cross section of any shape. This object can have conducting, dielectric or permeable properties. The main questions that we want to answer are:

- What does propagation represent?

- What do displacement and induced currents represent?

- What is the influence of permittivity, permeability and conductivity on these quantities and phenomenon?

- What means the cutoff phenomenon?

The first section of the paper deals with the solving method. The graphical interface of Depind is then described in the second section. The ability of Depind to help to understand the complex scattering phenomena is finally illustrated with several examples: radiation from a wire (in order to understand propagation phenomena), scattering by a lossy dielectric cylinder (in order to compare induced and displacement currents) and scattering by a parallel plate waveguide (in order to understand the cutoff phenomenon).

\section{The SOLVING MeTHOD}

For such an application, local quantities are required to display the results. On the other hand no analytical solution is available, especially in the case of lossy dielectrics. The solution is then obtained by computing the $2 \mathrm{D}$ wave equation using the $\mathrm{FE}$ method coupled either with a second order Bayliss-Turkel absorbing boundary condition (ABC) or with a boundary element method. Since an instant feedback to any data input is required, the solving time has to be minimized as much as possible. There is actually no particular difficulty in applying the FEM for such 
problems. The key-point lies in the optimization of the method: for CAE considerations, a great accuracy in the displayed results is not necessary; on the other hand, fast solving time is required for making the solving transparent for the user.

\section{A. The Numerical Solving}

The 2D wave equation is written either in TE mode for the $z$-component of the electric field $E_{z}$ or in TM mode for the $z$-component of the magnetic field $H_{z}$. For example, the Galerkin FE form for the TM mode is [3]:

$$
\begin{gathered}
\int_{\Omega}\left[\nabla W \cdot \frac{1}{j \omega \varepsilon} \nabla H_{z}+j \omega \mu W \cdot H_{z}\right] d \Omega \\
-\frac{1}{j \omega \varepsilon} \int_{\Gamma} W \cdot \frac{\partial H_{z}}{\partial n} d \Gamma=\langle\text { sources }\rangle
\end{gathered}
$$

where $W$ is the weighting function and the sources can be a current density or a plane wave. As results, depending on the studied mode of propagation, either the complex value of the normal component of the electric field $E_{z}$ or the normal component of the magnetic field $H_{z}$ are directly computed. The tangential dual field is obtained by derivation.

The main difficulty when solving unbounded wave problems with the FEM is the truncation of the infinite domain with a fictitious boundary. Two types of boundary conditions may then be employed on this outer boundary [4]. Transparent conditions, such as the BEM, are exact and allow in principle to locate the outer boundary as close as possible to the modeled device. But, since they are global, the resulting boundary matrix is full and nonsymmetric. On the other hand, ABC's preserve the sparsity of the FE system matrix, since they are only local. But they are built up as an approximation of the exact solution: they are not totally absorbing. Furthermore, a minimal distance between the boundary and the microwave device is necessary in order to get reasonably accurate results. Generally, a distance equal to one wavelength is recommended.

$A B C$ 's seem then to be well designed for our CAE purpose, since a high solving speed is required. However, they do not allow to decrease widely the frequency, since the size of the studied domain becomes rapidly small compared to the wavelength. For this reason both conditions have been implemented: BEM is used when the wavelength is large compared to the size of the domain (study of the wave radiated from a wire), while $\mathrm{ABC}$ is used when the size of the domain is comparable to the wavelength (study of the effect of the characteristics of the dielectric in scattering problems). The second order Bayliss-Turkel condition has been chosen in this case, leading to a circular outer boundary.

\section{B. Coupling with ABC for Scattering Problems}

The coupling is performed by inserting the second order Bayliss-Turkel ABC in the line integral of (1). In the case of scattering by an incident plane wave, the final F.E. form for the TM mode is:

$$
\begin{aligned}
\int_{\Omega} & {\left[\nabla W \cdot \frac{1}{j \omega \varepsilon} \nabla H_{z}+j \omega \mu W \cdot H_{z}\right] d \Omega } \\
& \quad-\frac{\alpha}{j \omega \varepsilon} \int_{\Gamma} W \cdot H_{z} d \Gamma+\frac{\beta}{j \omega \varepsilon} \int_{\Gamma} \frac{\partial W}{\partial \tau} \cdot \frac{\partial H_{z}}{\partial \tau} d \Gamma
\end{aligned}
$$

$$
\begin{aligned}
= & \frac{1}{j \omega \varepsilon} \int_{W} W \cdot \frac{\partial H_{z}^{i}}{\partial n} d \Gamma-\frac{\alpha}{j \omega \varepsilon} \int_{\Gamma} W \cdot H_{z}^{i} d \Gamma \\
& +\frac{\beta}{j \omega \varepsilon} \int_{\Gamma} \frac{\partial W}{\partial \tau} \cdot \frac{\partial H_{z}^{i}}{\partial \tau} d \Gamma
\end{aligned}
$$

with

$$
\alpha=-\frac{5-8 k^{2} R^{2}-12 j k R}{8 R(1-j k R)}
$$

and

$$
\beta=\frac{1}{2 R(1-j k R)},
$$

and $H_{z}^{i}$ is the incident field, $\Gamma$ is the boundary of the studied domain $\Omega$, and $R$ is the radius of $\Gamma$.

\section{Coupling with B.E.M.}

In $2 \mathrm{D}$, the magnetic field in free space may be computed using the integral equation:

$$
H_{z}=\int_{\Gamma}\left[G \frac{\partial H_{z}}{\partial n}-\frac{\partial G}{\partial n} H_{z}\right] d \Gamma
$$

where $G=(j / 4) H_{0}^{(2)}(\mathrm{kr})$ and $(\partial G / \partial n)=(j k / 4) H_{1}^{(2)}(\mathrm{kr})$ are the Hankel functions of second kind.

The coupling between the FEM and the BEM is performed by eliminating the normal derivative of the magnetic field in (1) and (3), and by approximatively symmetrizing the resulting matrix [3]-[5]. In matrix form, this leads to:

$$
\begin{gathered}
\text { FEM: }[S] \cdot\left[H_{z}\right]-[T] \cdot\left[\frac{\partial H_{z}^{b}}{\partial n}\right]=\text { [sources] } \\
\text { BEM: }[U] \cdot\left[H_{z}^{b}\right]-[V] \cdot\left[\frac{\partial H_{z}^{b}}{\partial n}\right]=0
\end{gathered}
$$

\section{Coupling:}

$$
\begin{aligned}
& {[S] \cdot\left[H_{z}\right]-\frac{1}{2}\left(\left([T] \cdot[V]^{-1} \cdot[U]\right)+\left([T] \cdot[V]^{-1} \cdot[U]\right)^{T}\right)\left[H_{z}^{b}\right]} \\
& \quad=[\text { sources }]
\end{aligned}
$$

where $\left[H_{z}^{b}\right]$ denotes the field on the boundary.

\section{Optimization of the Implementation}

The optimization of the implementation is done in the same way as presented in [2]:

- definition of a symmetry,

- optimization of the meshing, depending on the range of input parameters,

- use of first order triangles

- solving using the Cholesky factorization with the nested dissection renumbering algorithm.

- Since the geometry of the problem is not modified, the structure of the mesh remains unchanged and the local submatrices are pre-computed and stored in memory.

Special attention has been paid to the computation of the BE part. The singular integration is performed numerically with five Gauss points. Because the argument of the Hankel functions depends on the propagation constant, the entire BE matrices are 
TABLE I

Computation Times (ON a 333 MHz Pentium II PC) For SEVERAL PRoblem SizES DEPENDING ON THE METHOD USED FOR THE TRUNCATION OF THE FE DOMAIN

\begin{tabular}{|c|c|c|c|c|}
\hline \begin{tabular}{|c|}
$\begin{array}{c}\text { Number of } \\
\text { nodes }\end{array}$ \\
\end{tabular} & $\begin{array}{c}\text { Number of } \\
\text { boundary elements }\end{array}$ & $\begin{array}{l}\text { Method for } \\
\text { truncation }\end{array}$ & $\begin{array}{l}\text { CPU time ( } \\
\text { frequency }\end{array}$ & $\begin{array}{l}\text { ns) when changing: } \\
\text { material properties }\end{array}$ \\
\hline \multirow{2}{*}{374} & \multirow{2}{*}{43} & BEM & 220 & 110 \\
\hline & & $\mathrm{ABC}$ & 160 & 160 \\
\hline \multirow{2}{*}{437} & \multirow{2}{*}{43} & BEM & 220 & 160 \\
\hline & & $\mathrm{ABC}$ & 170 & 170 \\
\hline \multirow{2}{*}{576} & \multirow{2}{*}{45} & BEM & 280 & 160 \\
\hline & & $\mathrm{ABC}$ & 220 & 220 \\
\hline \multirow{2}{*}{685} & \multirow{2}{*}{59} & BEM & 440 & 220 \\
\hline & & $\mathrm{ABC}$ & 220 & 220 \\
\hline \multirow{2}{*}{742} & \multirow{2}{*}{83} & $\overline{B E M}$ & 770 & 330 \\
\hline & & $\mathrm{ABC}$ & 280 & 280 \\
\hline \multirow{2}{*}{799} & \multirow{2}{*}{91} & BEM & 850 & 380 \\
\hline & & $\mathrm{ABC}$ & 280 & 280 \\
\hline
\end{tabular}
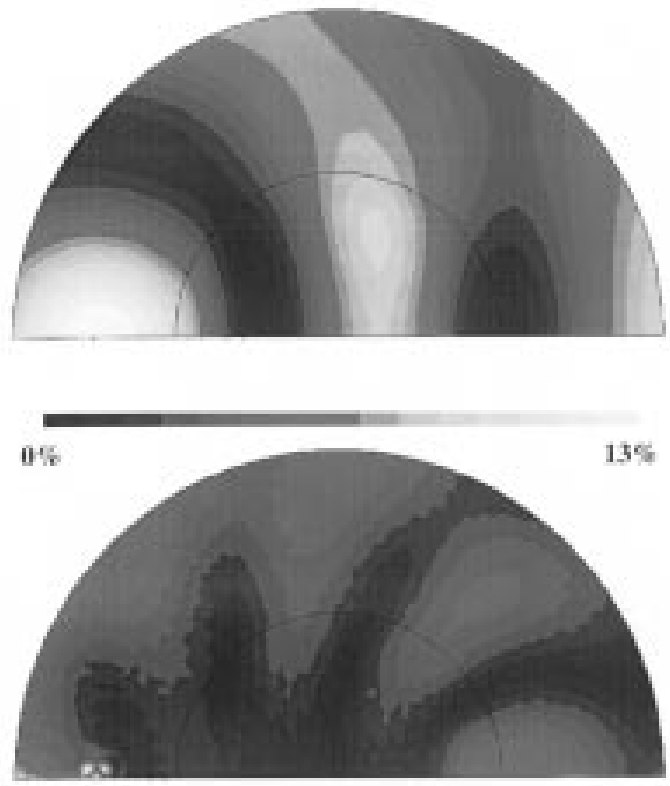

Fig. 1. 2D computation of the electric field radiated by a wire in presence of a dielectric cylinder $\left(\varepsilon_{r}=2, f=3 \mathrm{GHz}\right)$, with a symmetry plane. Coupling with the BEM. Top: magnetic field at the instant $t=0^{\circ}$. Bottom: error between both CAE and regular FE solution: max. error is about 13\%-located around the source-, and average error is $2.8 \%$.

re-computed-including inversion of $[V]$ and matrix multiplication-when the frequency is modified. On the other hand, when modifying the characteristics of the dielectric, only the FE part of the corresponding region is changed, leading to lower computation times. As shown in Table I, these tricks lead to solving times on a PC compatible with a smooth animation.

\section{E. Accuracy of the Results}

Several tests have shown the good accuracy of the developed method (Fig. 1). Comparisons have been made either with an analytical solution if possible, or with a regular finite element code developed for CAD purpose [5]. In all cases the averaged error on the studied domain is less than 5\%, validating the approximations which have been made. The main objective has

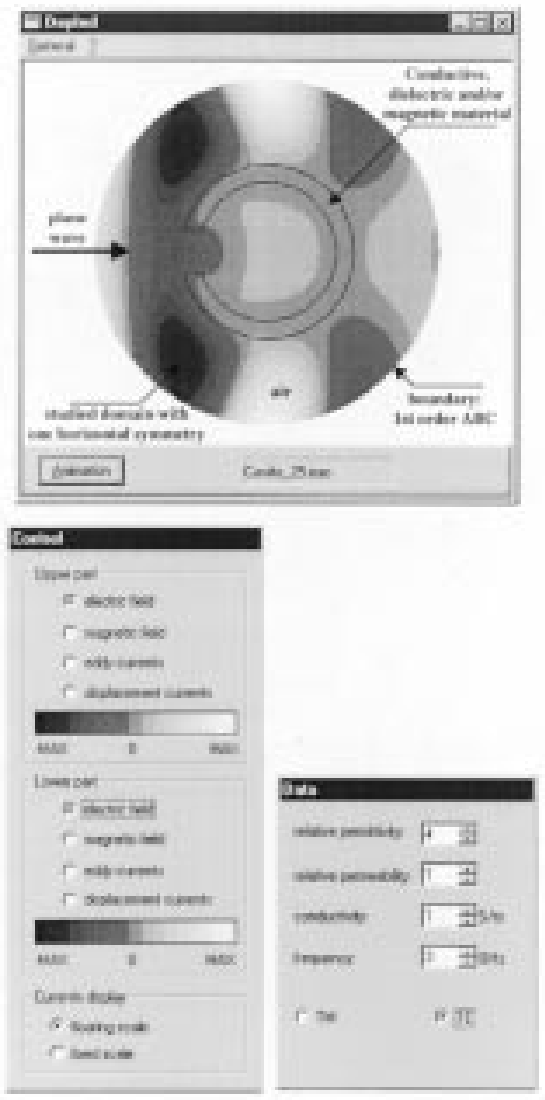

Fig. 2. Main, control and data windows. Circular cavity with an aperture.

been attained: the solving is sufficiently fast to be invisible for the user, and the computed results are sufficiently accurate.

\section{THE GRAPHICAL INTERFACE}

Using the data window, the frequency of the incident wave, and the values of conductivity, permittivity and permeability of the scatterer are chosen (Fig. 2). Either a transverse magnetic (TM) or a transverse electric (TE) mode can be defined. Different geometries are loaded from separate files: a circular cylinder (685 nodes), a square cylinder (700 nodes), a circular cavity with an aperture (684 nodes), a parallel plate waveguide (435 nodes), a wedge (610 nodes) are available.

As results, the eddy current density, the displacement current density, the electric field or the magnetic field can be visualized using animated color shading. In order to compare them, each of these quantities may be displayed independently in the upper and in the lower symmetric part of the studied domain. The time-animation of the solution allows a better understanding of the phenomena: at each time step, a new bitmap is defined according to the complex solution and is immediately displayed using a double-buffer.

\section{EXAMPLES OF APPLICATION}

\section{A. Electromagnetic Propagation Phenomenon}

This example (Fig. 3) is used in order to show the effect of the frequency of the current in a wire on the propagation 

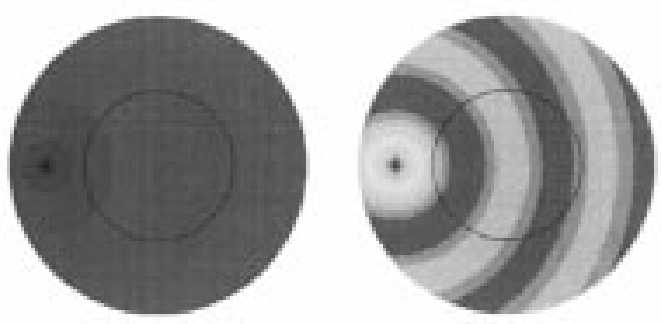

Fig. 3. Radiation from a wire in free space. Comparison of two propagation frequencies.
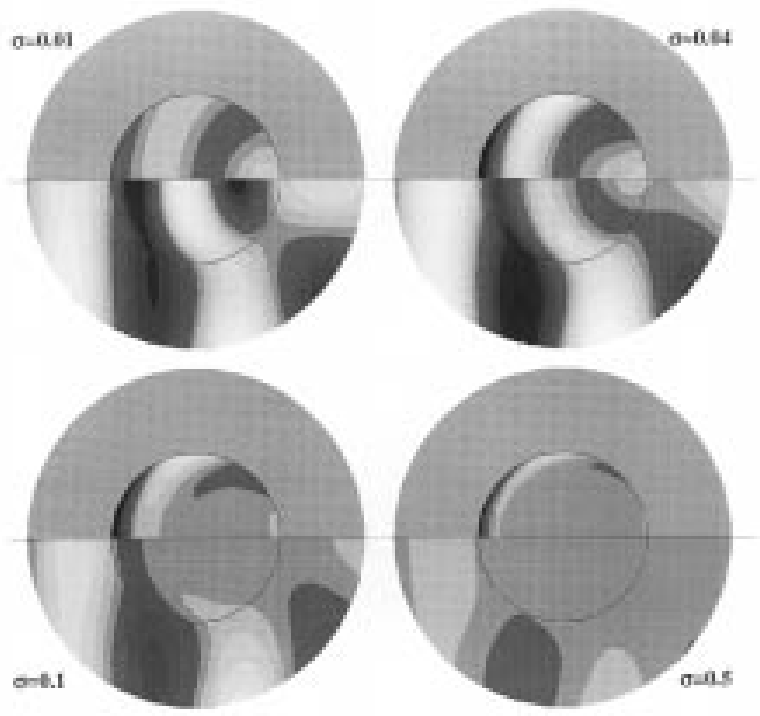

Fig. 4. Scattering by a lossy dielectric cylinder $\left(\varepsilon_{r}=3\right)$. Comparison of eddy current (upper part) and displacement current (lower part) densities for severa values of the conductivity of the dielectric cylinder.

phenomena. The range of the electromagnetic characteristics of the inner circle may vary from 1-10 for the relative permittivity and permeability, and from $0-10$ for the conductivity. Since the frequency can vary from $30 \mathrm{MHz}$ to $5 \mathrm{GHz}$ with a circular outer domain of radius 0.1 meter, the coupling between the FEM and the BEM is used.

\section{B. Induced and Displacement Currents}

One major goal of Depind is to understand the difference between eddy currents and displacement currents appearing when an object is illuminated with an electromagnetic wave. The studied problem is the scattering of an incident plane wave by an infinite circular cylinder at normal incidence. Depending on the relative values of the conductivity and the permittivity, and on the value of the frequency of the incident wave, the cylinder may be seen as (Fig. 4):

- Good conductor: the eddy current density is much larger than the displacement current density, and the notion of skin depth may then be shown (Fig. 4, bottom right).

- Good dielectric: there is no eddy current, and the total current density is approximately equal to the displacement

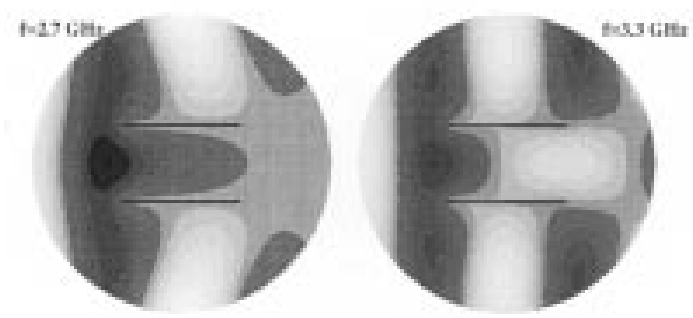

Fig. 5. Scattering by a parallel plate waveguide in normal incidence. Left: below the cutoff frequency. Right: above the cutoff frequency.

current density. The wavelength in the dielectric is reduced in the square root ratio of the permittivities.

- Absorbing material: both eddy current and displacement current densities are superimposed. They are in phase opposition. Eddy current prevent the development of displacement current inside the conducting body.

\section{Cutoff Phenomenon}

In order to illustrate this phenomenon, a parallel plate waveguide is illuminated by an incident plane wave propagating along its axis (Fig. 5). Depending on the value of the frequency, one can observe:

- Below the cutoff frequency, no propagation can occur inside the waveguide, and there is a rapid decay of the field inside the waveguide.

- Above the cutoff frequency: there is propagation inside the guide, which becomes more and more transparent to the incident wave when the frequency increases.

\section{CONCLUSION}

This CAE tool, based on graphical user interfaces, allows students and teachers to perform conceptual experiments on scattering phenomena. The user can work directly with several objects and change parameters values. He has an instantaneous answer through graphical display. This tool allows actually to perform virtual experiments. This is an important step to understand physical phenomena.

\section{REFERENCES}

[1] K. Preis, I. Bardi, O. Biro, R. Hoschek, M. Mayr, U. Peterlini, C. Magele, C. Paul, W. Renhart, and I. Ticar, "Computer animation of Electromagnetic phenomena,", http://www-igte.tu-graz.ac.at/index.htm.

[2] F. Buret, D. Muller, and L. Nicolas, "C.A.E. for magnetostatics," IEEE Trans. on Education, vol. 42, no. 1, pp. 45-49, Feb. 99.

[3] P. S. Shin, K. A. Connor, and S. J. Salon, "The hybrid finite elementboundary element solutions of waveguide problems," IEEE Trans. on Magnetism, vol. 25, no. 5, pp. 3943-3945, Sep. 1989.

[4] J. L. Yao Bi, L. Nicolas, and A. Nicolas, "Vector absorbing boundary conditions for nodal or mixed finite elements," IEEE Trans. on Magnetism, vol. 32, no. 3, pp. 848-853, May 96.

[5] - , "2D electromagnetic scattering by simple shapes: A quantification of the error due to open boundary," IEEE Trans. on Magnetics, vol. 29, no. 2, pp. 1830-1834, March 93. 